\title{
ADVANCED SPACE-BASED DETECTORS
}

David Cardimona

17 Jul 2014

Final Report

APPROVED FOR PUBLIC RELEASE; DISTRIBUTION IS UNLIMITED.

\author{
AIR FORCE RESEARCH LABORATORY \\ Space Vehicles Directorate \\ 3550 Aberdeen Ave SE \\ AIR FORCE MATERIEL COMMAND \\ KIRTLAND AIR FORCE BASE, NM 87117-5776
}




\section{DTIC COPY NOTICE AND SIGNATURE PAGE}

Using Government drawings, specifications, or other data included in this document for any purpose other than Government procurement does not in any way obligate the U.S. Government. The fact that the Government formulated or supplied the drawings, specifications, or other data does not license the holder or any other person or corporation; or convey any rights or permission to manufacture, use, or sell any patented invention that may relate to them.

This report was cleared for public release by the 377 ABW Public Affairs Office and is available to the general public, including foreign nationals. Copies may be obtained from the Defense Technical Information Center (DTIC) (http://www.dtic.mil).

\section{AFRL-RV-PS-TR-2014-0010 HAS BEEN REVIEWED AND IS APPROVED FOR PUBLICATION IN ACCORDANCE WITH ASSIGNED DISTRIBUTION STATEMENT}

//SIGNED//

DAVID CARDIMONA

Program Manager

//SIGNED//

BENJAMIN M. COOK, Lt Col, USAF

Deputy Chief, Spacecraft Technology Division

Space Vehicles Directorate
//SIGNED//

PAUL D. LEVAN, Ph.D.

Technical Advisor, Space Based Advanced Sensing and Protection

This report is published in the interest of scientific and technical information exchange, and its publication does not constitute the Government's approval or disapproval of its ideas or findings. 


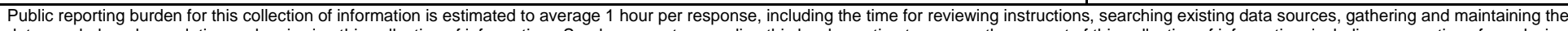

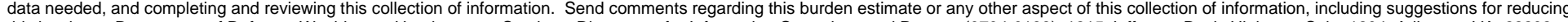

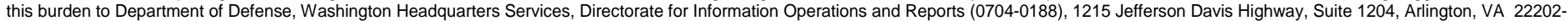

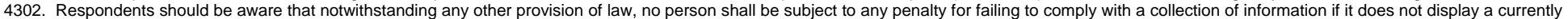
valid OMB control number. PLEASE DO NOT RETURN YOUR FORM TO THE ABOVE ADDRESS.

\begin{tabular}{|c|c|c|}
\hline $\begin{array}{l}\text { 1. REPORT DATE (DD-MM-YY) } \\
17-07-2014\end{array}$ & $\begin{array}{l}\text { 2. REPORT TYPE } \\
\text { Final Report }\end{array}$ & $\begin{array}{l}\text { 3. DATES COVERED (From - To) } \\
1 \text { Oct } 2005-30 \text { Jan } 2014\end{array}$ \\
\hline
\end{tabular}

17-07-2014 Final Report

4. TITLE AND SUBTITLE

1 Oct 2005 - 30 Jan 2014

Advanced Space-Based Detectors

5a. CONTRACT NUMBER

5b. GRANT NUMBER

5c. PROGRAM ELEMENT NUMBER

62601F

6. AUTHOR(S)

David Cardimona

5d. PROJECT NUMBER

4846

5e. TASK NUMBER

PPM00004410

5f. WORK UNIT NUMBER

EF002011

7. PERFORMING ORGANIZATION NAME(S) AND ADDRESS(ES)

8. PERFORMING ORGANIZATION REPORT NUMBER

Air Force Research L aboratorv

Space Vehicles Directorate

3550 Aberdeen Ave., SE

AFRL-RV-PS-TR-2014-0010

Kirtland AFB, NM 87117-5776

9. SPONSORING I MONITORING AGENCY NAME(S) AND ADDRESS(ES)

10. SPONSOR/MONITOR'S ACRONYM(S)

AFRL/RVSS

11. SPONSOR/MONITOR'S REPORT NUMBER(S)

\section{DISTRIBUTION I AVAILABILITY STATEMENT}

Approved for public release; distribution is unlimited. (Clearance 377ABW-2014-0319, dtd 6May2014)

\section{SUPPLEMENTARY NOTES}

\section{ABSTRACT}

At the Air Force Research Laboratory, Space Vehicles Directorate, Advanced Electro-Optical Space Sensors (AEOSS) Group, we are interested in improving the performance of, or modifying the capabilities of, infrared detectors in order to locate and identify dim and/or distant objects in space for surveillance and situational awareness missions. In this project we studied different possibilities that will address the requirements for operation in space (low temperatures, low photon backgrounds, long distances, high radiation).

\section{SUBJECT TERMS}

Detectors, Infrared, Quantum Dot Detectors, Quantum Well Detectors, Superlattice Detectors

\begin{tabular}{|c|c|c|c|c|c|}
\hline \multicolumn{3}{|c|}{ 16. SECURITY CLASSIFICATION OF: } & \multirow{2}{*}{$\begin{array}{l}\text { 17. LIMITATION } \\
\text { OF ABSTRACT } \\
\text { Unlimited }\end{array}$} & \multirow{2}{*}{$\begin{array}{l}\text { 18. NUMBER } \\
\text { OF PAGES } \\
\qquad 22\end{array}$} & \multirow{2}{*}{$\begin{array}{l}\text { 19a. NAME OF RESPONSIBLE PERSON } \\
\text { David Cardimona } \\
\text { 19b. TELEPHONE NUMBER (include area } \\
\text { code) }\end{array}$} \\
\hline $\begin{array}{l}\text { a. REPORT } \\
\text { Unclassified }\end{array}$ & $\begin{array}{l}\text { b. ABSTRACT } \\
\text { Unclassified }\end{array}$ & $\begin{array}{l}\text { c. THIS PAGE } \\
\text { Unclassified }\end{array}$ & & & \\
\hline
\end{tabular}


(This page intentionally left blank) 


\section{Table of Contents}

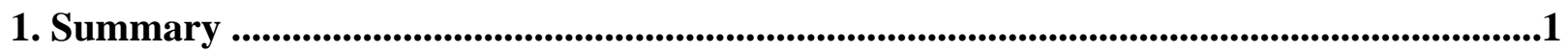

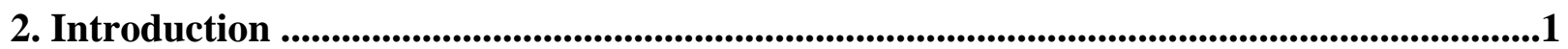

3. Methods, Assumptions, and Procedures .............................................................................2

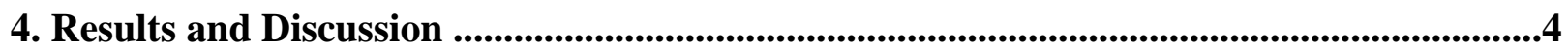

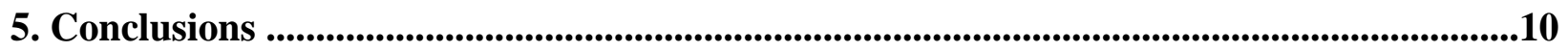

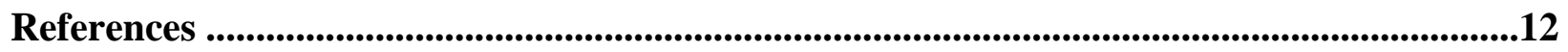

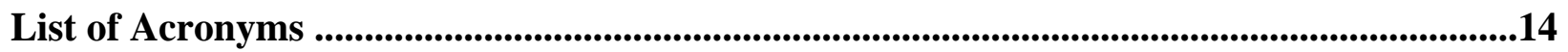


(This page intentionally left blank) 


\section{Summary}

During the early years of this project, 2006 - 2009, the Advanced Electro-Optical Space Sensors (AEOSS) Group was without an experimental contingent of researchers, due to personnel losses. During those years, our research efforts were in the theoretical physics that could be applied to improving the performance of detectors. Some of the topics of research during those years were: carrier transport in semiconductors; optical and electronic possibilities of graphene; singlephoton detection properties of surface acoustic waves; electromagnetic (EM) enhancement via quantum interference effects in photonic crystal cavities and via plasmonic interactions.

In 2010, we were fortunate to hire two new experimentalists, and in 2011 we were able to hire another. From this point until the end of this project in 2013, our research efforts covered both theoretical and experimental aspects of detector performance improvement. Our main areas of experimental research were in multi-color detectors and in radiation effects on those detectors. Our theoretical research continued along some of the above avenues, as well as attempting to explain some of the experimental results.

\section{Introduction}

At the Air Force Research Laboratory, Space Vehicles Directorate, Advanced Electro-Optical Space Sensors Group, we are interested in improving the performance of, or modifying the capabilities of, infrared (IR) detectors in order to locate and identify dim and/or distant objects in space for surveillance and situational awareness missions. Our primary interests are in observations of objects both on earth and in space, each of which has very different background requirements. In addition, the space environment itself is especially demanding of any sensor system that will be expected to work continuously for long periods of time in such a challenging environment. In this project we studied different possibilities that will address the requirements for operation in space (low temperatures, low photon backgrounds, long distances, high radiation). 


\section{Methods, Assumptions, and Procedures}

In our experiments, the devices studied were mounted and wire-bonded to a 68 pin leadless chip carrier (LCC) for testing. The LCC was fastened to the cold-finger of a pour-filled cryogenic dewar capable of reaching $4 \mathrm{~K}$. The dewar was pumped down to a vacuum of $5 \times 10^{-7}$ Torr and cryogenically cooled to $77 \mathrm{~K}$ with liquid nitrogen. The dark current measurements were taken with a cold shield surrounding the device, which was also cooled to $77 \mathrm{~K}$, blocking nearly all IR radiation. Two Keithley Model 236 Source Measurement Units were used to apply the sourcedrain and gate voltages. A Lakeshore Model 340 Temperature Controller monitored the temperature of the detector via a diode fastened to the cold finger. Source-drain voltages from $1.0 \mathrm{~V}$ to $1.0 \mathrm{~V}$ were applied across the device while simultaneous current measurements were made. The gate voltages ranged from $0.0 \mathrm{~V}$ to $-0.8 \mathrm{~V}$.

The dark current in the devices were measured at $77 \mathrm{~K}$ with the direct-current (DC) source-drain voltage being stepped in $1.0 \mathrm{mV}$ increments from - $1.0 \mathrm{~V}$ to $1.0 \mathrm{~V}$. The absolute value of the measured source-drain (lateral) current $\mathrm{I}_{\mathrm{SD}}$ was plotted with respect to the source-drain voltage $\mathrm{V}_{\mathrm{SD}}$. This measurement was repeated at different gate voltages $\mathrm{V}_{\text {Gate }}$.

A similar sample mounting scheme was used for taking differential conductance measurements. Here, a small $13 \mathrm{~Hz}, 100 \mu \mathrm{V}$ AC signal, output from a Stanford Research Systems Model SR850 DSP Lock-In Amplifier, was passed to an external, ambient temperature, $10 \mathrm{M} \Omega$ resistor connected in series to the drain contact of the device, with its source contact grounded. This setup allows the differential conductance of the device to be measured via low frequency lock-in measurements of the voltage across the $10 \mathrm{M} \Omega$ resistor. Measurements of the differential conductance were also taken as the gate voltage was swept from $0.0 \mathrm{~V}$ to $-0.85 \mathrm{~V}$.

Simple DC photocurrent measurements were taken with a cold shield, cooled to $77 \mathrm{~K}$, in place and having a hole cut in one side to allow for incident $300 \mathrm{~K}$ blackbody radiation. The radiation passed into the dewar through a KRS5 window, leading to approximately f/2 optical alignment.

During this project, we upgraded our spectral response measurement system, a Nicolet Magna

Approved for public release; distribution is unlimited. 
860 Fourier Transform IR (FTIR) spectrometer. The FTIR spectral response setup was upgraded to allow for step-scan/lock-in style measurements, which allow very sensitive spectral response measurements to be performed.

In the radiation studies of the last few years, the detectors for these experiments were all irradiated at the Crocker Nuclear Laboratory (CNL) Radiation Effects Measurement and Test Facility at the University of California, Davis. The facility uses a beam line from the 76" isochronous cyclotron at CNL to provide protons with energies up to $68 \mathrm{MeV}$. The beam profile at the test position varies by less than $3 \%$ radially outward to $2 \mathrm{~cm}$, providing a nearly uniform beam for irradiation.

Each experiment was also done using a similar step-wise irradiation-measurement approach of measuring the detectors, dosing with protons, re-measuring, re-dosing, etc. until a Total Ionizing Dose $(\mathrm{TID})>100 \mathrm{kRad}(\mathrm{Si})$ was reached. This step-wise approach best allowed for degradation rates to be determined since the changes in dark current are often not perfectly linear across an entire fluence range. Irradiation was performed for every experiment with $63 \mathrm{MeV}$ protons across a similar fluence range $\left(10^{9}-10^{12} \mathrm{p} / \mathrm{cm}^{2}\right)$ using roughly similar dose schedules (e.g. 2, 5, $10,20,50,100 \mathrm{kRad}(\mathrm{Si}))$. At this proton energy, the Bragg peak is expected to be well past the active region of the detectors. Thus, a nearly uniform dose and induced damage across the thickness of the active region was expected. The detectors were all held at their operating bias voltage and temperature during irradiation. Typically the operating voltage is in the millivolt range where the dark current tends to remain diffusion-limited and the photocurrent has roughly leveled off. The measurements taken before and after each dose step consisted of dark-current and photocurrent measurements from various sized $(\sim 15-800 \mu \mathrm{m})$ detectors on the same process evaluation chip (PEC). The dark current was measured using a pair of DC sourcemeasure units with a cooled shutter held at $77 \mathrm{~K}$ in front of the aperture, blocking nearly all the irradiance on the detectors on the PEC. This dual instrument measurement scheme allowed for the dark current path through each detector to be confirmed. The optical measurements were all taken using flood illumination from a well characterized blackbody source passing through an optical chopper, a narrow band filter $(3-3.5 \mu \mathrm{m})$ well within the linear absorption regime of the detectors and a $4 \mathrm{~mm}$ aperture located inside the dewar and held at $77 \mathrm{~K}$ at a fixed distance from 
the detector, producing an $\mathrm{f} / \# \sim 39$. This approach produced a known irradiance level on the detector reflective of strategic or other applications in the space environment. It also allowed for the photocurrent to be very accurately measured using an external transimpedance amplifier coupled to a lock-in amplifier referred to the chopper reference signal. Flood illumination has the advantage over focused illumination of allowing for both the bulk quantum efficiency and a lateral optical collection length to be determined.

\section{Results and Discussion}

To improve detector or electronic device performance, we investigated the electronic transport and photonic properties of a two-dimensional electron gas (2DEG) such as that found at a heterojunction between the semiconductor composed of galium and arsenide (GaAs) and one composed of aluminum, galium, and arsenide (AlGaAs). Related physical properties of narrow quantum wires were also the subject of theoretical investigations by us because of their potential for device applications in the field of nanotechnology. [1]

Graphene is an emerging material that could change the way electronic components are made, and could help computing performance continue to grow. Graphene can be described as a oneatom thick layer of graphite. High-quality graphene is strong, light, nearly transparent, and an excellent conductor of heat and electricity. Experimental results from transport measurements show that graphene has a remarkably high electron mobility at room temperature. Electrons move through graphene incredibly fast and exhibit behavior as if they are massless. Graphene's unique optical properties produce an unexpectedly high opacity for an atomic monolayer. Recent advances in the fabrication techniques of graphene nanoribbons (GNRs), together with the long electron mean free path found in GNRs, have stimulated considerable interest in their potential applications as interconnects in nano circuits. The optical response of graphene nanoribbons is tunable into the terahertz $(\mathrm{THz})$ regime by an applied magnetic field. For these reasons, we intensively studied several theoretical aspects of this new material system. [2,3]

Approved for public release; distribution is unlimited. 
A surface acoustic wave (SAW) is an acoustic wave that travels along the surface of a material exhibiting elasticity, with an amplitude that decays exponentially with depth into the substrate. About 18 years ago, a method was introduced by which SAWs could be used to generate quantized electrical currents. In these experiments, a SAW was produced by applying a radiofrequency signal to an interdigitated transducer. The SAW propagates across a GaAs surface until it reaches a region of GaAs/InGaAs (InGaAs is indium/gallium/arsenide) heterostructure containing a quantum well layer of a 2DEG. In this 2DEG region the SAW is heavily screened, but its associated electrostatic wave is still capable of dragging charge with it. In the center of the device, a metallic split-gate or etched-trench gate is used to create a narrow depleted channel with a lateral confinement energy. As each trough of the SAW approaches the depleted channel, the SAW amplitude is increased. The combined SAW and split-gate potentials can become capable of producing a one-dimensional (1D) quantum-dot-like confinement potential. The observed current quantization arises from each dot capturing an equal number of electrons in this process. The accuracy of the current quantization is close to one part in $10^{4}$. SAW-based singlephoton emission techniques have now been established at low temperatures. Our interest was in the opposite physical mechanism: single-photon detection. The potential of the SAW-based optoelectronic device lies in its ability to detect radiation down to a single photon. The exploitation of SAWs to security screening and detection of IR signals was an appealing idea. The acousto-electric detectors, including in the visible and IR, are expected to be very robust against vibrations, mechanical stresses, and EM interference. These properties, in addition to high sensitivity and small size, made them attractive for applications such as the security of airborne and space-borne objects, like aircraft and satellites. For these reasons, we pursued this topic theoretically for a short while. [4]

To see dim/distant objects, we theoretically investigated two optical signal amplification schemes. One of the schemes used quantum interference and electromagnetically-induced transparency (EIT) in quantum dots surrounded by a photonic crystal $(\mathrm{PhC})$ cavity to detect weak signals. The EIT provides the detection mechanism and the $\mathrm{PhC}$ cavity provides the extreme sensitivity. We first considered a quantum dot with a three-dimensional quantum confinement. The energy gap between the valence and conduction bands is determined by the host material for the quantum dot. The energy separation between two adjacent sublevels in the conduction or the 
valence band is determined by the size of the quantum dot. By designing a quantum dot to have two sublevels in the conduction band, we could use the principle of EIT to create a scheme for weak-signal (perhaps even single-photon) detection. We first apply a tunable visible laser (the 'probe' laser) to couple the top-most sublevel in the valence band of the quantum dot to the second sublevel in the conduction band. In this case, there will be a strong interband absorption associated with this probe laser. If a second IR light beam (the coupling light - which, in our case, is the weak signal to be detected) is then incident on this quantum dot at the same time with a photon energy in resonance with the energy separation between the first and the second sublevels in the conduction band, the absorption of the probe laser will go to zero due to the EIT, which can then be detected. The dephasing of the induced optical coherence due to carrier-carrier and carrier-phonon scattering is the biggest roadblock to the realization of EIT in a quantum-dot system. If the Rabi coupling between the first and the second sublevels in the conduction band by the coupling signal light is very strong compared to the dephasing rate, the EIT becomes robust to the dephasing of the optical coherence. The enhancement of this Rabi coupling can be obtained by employing a PhC cavity. [5]

Photonic crystals are to EM fields as semiconductor materials are to electrons. Just as semiconductor materials have forbidden energy bandgaps due to the periodic arrangement of many atoms, a material with periodic variations in the dielectric constant can lead to a gap in the frequencies of EM radiation allowed to exist and propagate in the structured material, a so-called photonic bandgap (PBG). A three-dimensional (3D) PhC consists of dielectric variations in all dimensions, and will produce a PBG for light incident from any direction. Once a material with a PBG has been created, it is possible to introduce a defect into the material (by subtracting or adding dielectric material at one spot), analogous to acceptor or donor defects in semiconductors. Similar to how these semiconductor defects introduce energy levels into the forbidden bandgap, the defect in a PhC can be designed with any frequency in the PBG, thereby allowing EM radiation at that frequency to exist and propagate in the crystal. Because the photonic defect is surrounded by a material with a PBG, the field should experience little or no loss, similar to being in a high-Q cavity. Thus, defects within PBG crystals can be considered microcavities. The introduction of quantum dots into the PhC cavity will greatly increase the Rabi dipole coupling (on the order of one million times) by reducing the optical-mode volume, thereby allowing the

Approved for public release; distribution is unlimited. 
signal field to be extremely weak while still providing a strong coupling. At the same time, the spectrum of the broad-band coupling signal will be squeezed into a very narrow frequency region in resonance with the first and second sublevels in the conduction band. [6]

The other amplification scheme we studied theoretically involved near-field enhancements due to surface-plasmon-polariton interactions on nanopatterned metal surfaces. A plasmon is the quasiparticle resulting from the quantization of plasma oscillations, which are density waves of the charge carriers in a conducting medium such as a metal, doped semiconductor, or plasma. Electron charges at a conductor-dielectric interface that undergo these coherent oscillations, are called surface plasmons. When these plasmons near surfaces are excited by an optical field, a polariton is the result. Polaritons are quasiparticles resulting from a strong coupling of EM waves with an electric dipole-carrying excitation. Thus, a polariton is the result of the mixing of a photon with an excitation of a material. Phonon-polaritons result from coupling an IR photon with a phonon. Exciton-polaritons result from coupling visible light with an exciton. Surfaceplasmon-polaritons (SPPs) result from coupling surface plasmons with light. Surface plasmons are typically excited at metal-dielectric interfaces. However, in a completely analogous fashion, they can be excited in a quantum-confined system where the metal is replaced with an electron sheet charge on a semiconductor. Light incident normal to the semiconductor surface will, in general, not excite a surface plasmon, owing to the need to conserve both energy and momentum in the excited system. The introduction of surface structures such as a grating, and the subsequent diffraction of the incident wave, allows for the generation of surface plasmons at wavelengths that are functions of both the grating period and the sheet charge density. In 1998, Ebbesen and co-workers reported an enhancement of the optical transmission through an array of deep sub-wavelength cylindrical holes on a silver film. Similar phenomena were observed in sub-wavelength metallic gratings. These enhanced optical transmissions are believed to be a result of light coupling to SPPs in structured metallic films. Our research efforts investigated this enhancement in anticipation of placing a photodetector material directly below the patterned metal, or doped semiconductor, surface. $[7,8]$

To identify dim/distant objects in space, we investigated a couple of multi-color detector schemes. For one of the multi-color schemes, we developed a new IR detector design, henceforth 
referred to as a lateral quantum dot IR photodetector (LQDIP), with the potential for a tunable spectral response. In this design, indium/arsenide InAs quantum dots are buried in a GaAs quantum well, which is in turn tunnel-coupled to a second GaAs quantum well. Photo-excited electrons from the quantum dots are expected to tunnel over to the second well, where they are then swept out via a lateral (perpendicular to the growth direction) bias voltage. The lateral photocurrent is in part directed to tunnel into the second quantum well by the depletion field of a narrow pinch-off gate, applied vertically (parallel to the growth direction). Under the proper biasing arrangement, this detector architecture is expected to exhibit the ability to tune to select IR frequencies as well as operate with reduced dark currents and unity gain in the second well. [9]

Infrared detectors operated in the space environment are required to have high performance while being subjected to a variety of radiation effects. For space-based imaging systems, radiation tolerance to both displacement damage and total ionizing dose (TID) radiation effects continues to be a major performance concern. Sources of radiation in space include the trapped particles in the Van Allen belts and transient events such as solar events and galactic cosmic rays. When properly passivated, conventional mercury-cadmium-telluride (MCT) -based IR detectors have been shown to perform well in space environments. However, the inherent manufacturing difficulties associated with the growth of MCT has resulted in a research thrust into alternative detector technologies. Over the last several years the development of type-II Strained Layer Superlattice (SLS) IR photodetectors has yielded devices that may offer plausible alternative technology to conventional MCT-based photodetectors. Prevailing theory predicts that SLS-based detector technologies will have several potential advantages over MCT technologies, including lower dark currents and higher operating temperatures, due to a suppression of the Auger recombination mechanism. However, experimentally it has been found that conventional p-on-n and n-on-p SLS detectors (where "n" and "p" refer to negative and positive doping of the semiconductor junction) have high dark current and thus, do not reach theoretically predicted performance benchmarks. The two prevailing contributors to this high dark current are the generation-recombination (GR) current due to trap-assisted tunneling in the depletion region and surface leakage currents resulting from a lack of fully passivated mesa sidewalls. An emerging detector architecture, complementary to the SLS detector structure, 
utilizes a unipolar single-band barrier design, henceforth referred to as $\mathrm{nBn}$ (two negativelydoped regions separated by an undoped barrier region), which effectively blocks majority carrier conduction, thereby reducing dark current to more acceptable levels, while keeping the inherent advantages of the SLS design. Specific advantages of SLS over MCT include wavelength tunability, improved uniformity and operability, potentially at a reduced manufacturing cost. In addition, long-wave IR detector technologies with the ability to operate at or near room temperature are very important for many civil and military applications including chemical identification, surveillance, defense, and medical diagnostics. Eliminating the need for cryogenics in a detector system can reduce cost, weight, and power consumption, as well as simplify the sensor system design and allow for widespread usage. It is anticipated that these detectors based on unipolar barrier designs will have an ability to lower dark current and increase a detector's operating temperature. We began to investigate this new field of research by performing experimental characterizations of the electrical and optical response of an $\mathrm{nBn}$ detector composed of n-type InAs/GaSb (GaSb is gallium/antimonide) SLS absorber (n) and contacts (n), with an Aluminum/Galium/Antimonide AlGaSb barrier (B). [10]

We combined our efforts in graphene and plasmonics to develop a self-consistent theory involving Maxwell's equations and a density-matrix linear-response theory. We investigated a doped graphene micro-ribbon array electromagnetically-coupled to a quantum well electron gas sitting at an interface between a half-space of air and another half-space of a doped semiconductor substrate, which supports a surface-plasmon mode. The coupling between a spatially-modulated total EM field and the electron dynamics in a Dirac-cone of a graphene ribbon, as well as the coupling of the far-field specular and near-field higher-order diffraction modes, were included in the electron optical-response function we derived. We found full analytical expressions with nonlocality for the optical-response functions of a two-dimensional electron gas and a graphene layer with an induced bandgap. Both the near-field transmissivity and reflectivity spectra, as well as their dependence on different configurations of our system and on the array period, ribbon width, graphene chemical potential of quantum well electron gas and bandgap in graphene, were studied. Moreover, the transmitted electric field intensity distribution was calculated to demonstrate its connection to the mixing of specular and diffraction modes of 
the total EM field. An externally-tunable EM coupling among the surface, conventional electrongas, and massless graphene intraband plasmon excitations was discovered and explained. [11] We also combined our plasmonic and scattering studies with our investigations of quantum interference in three-level systems. In this effort, both dynamic and static approaches were proposed and investigated for controlling the optical phase of a p-polarized light wave guided through a surface-patterned metallic structure with subwavelength features. For dynamic control, the important role of photo-excited electrons in a slit-embedded atomic system with fieldinduced transparency (FIT) was discovered within a narrow frequency window for modulating the intensity of focused transmitted light in the near-field region. This was facilitated by EM coupling to surface plasmons between the two FIT-atom embedded slits. The near-field distribution could be adjusted by employing a symmetric (or asymmetric) slit configuration and by a small (or large) slit separation. In addition, the cross-transmission of a light beam was also predicted as a result of this strong coupling between optical transitions in embedded FIT atoms and surface plasmons. For static control, the role of surface curvature was found for focused transmitted light passing through a Gaussian-shaped metallic microlens embedded with a linear array of slits. A negative light-refraction pattern, which is associated with higher-order diffraction modes, was found for large angles of incidence in the near-field region. This anomalous negative refraction could be suppressed when higher-order waveguide modes of light leak through a very thin film. In addition, this negative refraction could also be suppressed with a reinforced reflection at the left foothill of a Gaussian-shaped slit array of the forwardpropagating surface-plasmon wave at large angles of incidence. We predicted a near-field focusing of the light with its sharpness dynamically controlled by the frequency of the light in a very narrow window. [12]

\section{Conclusions}

The projects that will be continuing into the future are: attempting to make the LQDIP concept work - but at a reduced level and possibly for only another year, and investigating radiation effects in IR detectors at an increased level of effort. We intend to start an effort to theoretically investigate the origin, types, dynamics, and effects of defects in semiconductor materials and 
devices produced by exposure to high-energy particles. We will microscopically model the nuclear physics of the interactions in order to determine the defect atomic configuration, collision probabilities and differential cross-sections. This will give us the damage production rate, which we then will carry into a statistical modeling phase of the project. In this stage we will use a quantum statistical model to obtain both the vacancy and interstitial atom distribution functions. This will then be taken into a semiconductor device modeling stage where we will quantum-mechanically calculate the effects of defects on the physical processes within electronic devices, and determine methods of mitigation. The final objective of stage one will be to determine the selection of device materials and structures for improved next-generation performance. The objectives of stage two will be to determine what types of most active defects and associated electronic properties are in the devices, how many defects are generated, where these defects are distributed, and what damage effects these defects will have on electronic devices. The overall objective of the final stage of this project will be to design a possible system-level protection scheme for radiation damage. Particle radiation can damage electronic circuits and sensor systems in satellites, both commercial and other. This project will develop ways to mitigate that damage, thus saving the cost of replacing whole satellites in orbit. Indeed, any electronic or optoelectronic device in a high particle-radiation environment is susceptible to performance degradation. Hence, these studies will help prevent (or reduce at least) that degradation as well.

Approved for public release; distribution is unlimited. 


\section{References}

1. Lyo, S. K. and Huang, D. H., "Effect of electron-electron scattering on the conductance of a quantum wire studied with the Boltzman transport equation,” Phys. Rev. B 73, 205336 (2006).

2. Gumbs, Godfrey; Huang, Danhong, and Echenique, P. M., “Comparing the image potentials for intercalated graphene with a two-dimensional electron gas with and without a gated grating,” Phys. Rev. B 79, 035410 (2009).

3. Huang, Danhong; Gumbs, Godfrey, and Roslyak, O., "Field-enhanced electron mobility by nonlinear phonon scattering of Dirac electrons in semiconducting graphene nanoribbons,” Phys. Rev. B 83, 115405 (2011).

4. Huang, Danhong; Gumbs, Godfrey, and Pepper, M., “Effects of inelastic capture, tunneling escape, and quantum confinement on surface acoustic wave-dragged photocurrents in quantum wells,” J. Appl. Phys. 103, 083714 (2008).

5. Alsing, P. M.; Cardimona, D. A., and Huang, D. H., “Quantum interference near a photonic band edge beyond the weak-field approximation,” Phys. Rev A 76, 043802 (2007).

6. Cardimona, D. A.; Alsing, P. M.; Mozer, H., and Rhodes, C., "Interference effects in a three-level atom in a cavity beyond the weak-field approximation,” Phys. Rev. A 79, 063817 (2009).

7. Gumbs, Godfrey and Huang, Danhong, “Electronically modulated two-dimensional plasmons coupled to surface plasmon modes,” Phys. Rev. B 75, 115314 (2007).

8. Wellems, L. David; Huang, Danhong; Leskova, T. A., and Maradudin, A. A., "Nanogroove array on thin metallic film as planar lens with tunable focusing," Phys. Lett. A 376, pp. 216-220 (2012).

9. Guidry, D. H.; Morath, C. P.; Cowan, V. M., and Cardimona, D. A., "I-V and differential conduction characteristics of an AlGaAs/GaAs lateral quantum dot infrared photodetector” J. Electronic Materials 41, pp. 2679-2685 (2012).

10. Cowan, V. M.; Morath, C. P.; Hubbs, J. E.; Myers, S.; Plis, E., and Krishna, S., "Radiation tolerance characterization of dual band InAs/GaSb type-II strain-layer superlattice pBp detectors using 63 MeV protons,” Appl. Phys. Lett. 101, 251108 (2012).

Approved for public release; distribution is unlimited. 
11. Huang, Danhong; Gumbs, Godfrey, and Roslyak, Oleksiy, "Effects of nonlocal plasmons in gapped graphene micro-ribbon array and two-dimensional electron gas on near-field electromagnetic response in the deep subwavelength regime,” Appl. Opt. 52, pp. 755-769 (2013).

12. Huang, Danhong; Easter, M. Michelle; Wellems, L. David; Mozer, Henry; Gumbs, Godfrey; Cardimona, D. A., and Maradudin, A. A., "Dynamic and static control of the optical phase of guided p-polarized light for near-field focusing at large angles of incidence,” J. Appl. Phys. 114, 033106 (2013). 


\section{List of Acronyms}

1D - one-dimensional

2DEG - two-dimensional electron gas

$3 \mathrm{D}$ - three-dimensional

AEOSS - Advanced Electro-Optical Space Sensors

AlGaAs - Aluminum/Galium/Arsenide

AlGaSb - Aluminum/Galium/Antimonide

CNL - Crocker Nuclear Laboratory

DC - direct current

EIT - electromagnetically-induced transparency

EM - electromagnetic

FIT - field-induced transparency

FTIR - Fourier transform IR

GaAs - Galium/Arsenide

$\mathrm{GaSb}$ - gallium/antimonide

GNR - graphene nanoribbon

GR - generation-recombination

InAs - Indium/Arsenide

InGaAs - Indium/Galium/Arsenide

IR - infrared

LCC - leadless chip carrier

LQDIP - lateral quantum dot infrared photodetector

MCT - mercury-cadmium-telluride

$\mathrm{nBn}$ - negatively-doped material, barrier material, negatively-doped material

$\mathrm{PBG}$ - photonic bandgap

$\mathrm{PEC}$ - process evaluation chip

$\mathrm{PhC}$ - photonic crystal

SAW - surface acoustic wave

SLS - strained-layer superlattice

SPP - surface plasmon polariton

$\mathrm{THz}$ - terahertz

TID - total ionizing dose 


\section{DISTRIBUTION LIST}

DTIC/OCP

8725 John J. Kingman Rd, Suite 0944

Ft Belvoir, VA 22060-6218

$1 \mathrm{cy}$

AFRL/RVIL

Kirtland AFB, NM 87117-5776 2 cys

Official Record Copy

AFRL/RVSS/David Cardimona 1 cy 
(This page intentionally left blank)

Approved for public release; distribution is unlimited. 